\title{
Coronary artery perfusion and myocardial performance after patent ductus arteriosus ligation
}

\author{
Arvind Sehgal, MB, MSc, MRCPCH, ${ }^{\mathrm{a}, \mathrm{b}}$ and Patrick J. McNamara, MB, BCh, MRCPCH ${ }^{\mathrm{c}, \mathrm{d}}$
}

Objectives: To study coronary artery (CA) perfusion and myocardial performance after patent ductus arteriosus (PDA) ligation. The postoperative course in premature infants undergoing surgical ligation of PDA is often complicated by cardiorespiratory instability secondary to impaired left ventricular performance.

\begin{abstract}
Methods: Serial echocardiography was performed before and after (1, 8, and 24 hours) PDA ligation to assess systolic (left ventricular output [LVO]) and diastolic (isovolumic relaxation time, E and A wave peak velocity) myocardial performance, and CA diastolic flow (CA velocity time integral and flow). The ratio of CA flow to LVO was calculated as a surrogate of coronary flow.

Results: A total of 20 infants (gestational age at birth, $26.3 \pm 0.7$ weeks) requiring PDA ligation at a median of 28.5 days (range, 9-40) after birth and weight of $780 \mathrm{~g}$ (range, 570-2840) were studied. A postoperative increase in the CA flow/LVO ratio was demonstrated. An early decrease in $\mathrm{E}$ and A wave peak velocity $(P<.05)$ and increase in isovolumic relaxation time $(P<.05)$ were demonstrated at 1 hour, before any clinical deterioration. A low baseline CA velocity time integral was associated with a low E/A ratio $(r=0.63, P=.01)$ at 1 hour and lower systolic blood pressure at 8 hours $(r=0.5, P=.05)$. The postoperative need for inotropes $(\mathrm{n}=8)$ was associated with a low baseline CA velocity time integral at 1 hour $(\mathrm{r}=0.52, P<.05)$, low LVO at 1 and 8 hours $(P<.05)$, and increased oxygen requirement at 24 hours $(P<.05)$.
\end{abstract}

Conclusions: PDA ligation is followed by altered CA perfusion. Perioperative evaluation of the CA perfusion can help identify neonates at risk of impaired myocardial performance, systolic hypotension, and the need for inotropes. (J Thorac Cardiovasc Surg 2012;143:1271-8)

Hemodynamically significant patent ductus arteriosus (hsPDA) is seen in about $40 \%$ of extremely low-birthweight infants. ${ }^{1}$ One of the hallmarks of hsPDA is low diastolic blood pressure. Because coronary artery (CA) perfusion is dependent on the aortic diastolic pressure, premature infants with hsPDA might be susceptible to compromised CA flow and chronic myocardial ischemia. ${ }^{2}$ This hypothesis is supported by previous data demonstrating ST-segment depression on the electrocardiogram ${ }^{3}$ and elevations in plasma troponin ${ }^{4}$ that normalized after therapeutic intervention in premature infants with a hsPDA. We have previously reported impaired left ventricular (LV) systolic performance after PDA ligation. ${ }^{5}$ In that report, we speculated that the nature of the deterioration reflects an inability

\footnotetext{
From the Monash Newborn, ${ }^{\mathrm{a}}$ Monash Medical Centre and Monash University, ${ }^{\mathrm{b}}$ Victoria, Australia; Division of Neonatology and Department of Pediatrics, ${ }^{c}$ University of Toronto, Toronto, Ontario, Canada; and Physiology and Experimental Medicine Program, ${ }^{\mathrm{d}}$ Hospital for Sick Children, Toronto, Ontario, Canada Disclosures: Authors have nothing to disclose with regard to commercial support. Received for publication June 8, 2011; revisions received Sept 8, 2011; accepted for publication Oct 20, 2011; available ahead of print Dec 8, 2011.

Address for reprints: Patrick J. McNamara, MB, MSc, MRCP, MRCPCH, Division of Neonatology and Department of Pediatrics, University of Toronto, Physiology and Experimental Medicine Program, SickKids Research, Department of Neonatology, Hospital for Sick Children, 555 University Avenue, Toronto, ON M5G1X8 Canada (E-mail: patrick.mcnamara@ sickkids.ca).

$0022-5223 / \$ 36.00$

Copyright (c) 2012 by The American Association for Thoracic Surgery doi:10.1016/j.jtcvs.2011.10.035
}

of the immature myocardium to tolerate an increase in LV afterload. Chronic myocardial hypoperfusion secondary to low CA diastolic flow might increase the likelihood of impaired myocardial performance when subjected to the stressor of PDA ligation and altered loading conditions. The effect of hsPDA on coronary blood flow and its relationship to postoperative myocardial performance remains unknown.

\section{METHODS}

\section{Study Design}

A prospective observational study of premature infants undergoing PDA ligation at an outborn quaternary neonatal intensive care unit (NICU).

\section{Study Objective}

The primary objective was to characterize the trends in CA blood flow after PDA ligation. The secondary objective was to investigate the relationship between the CA blood flow and indexes of myocardial performance after PDA ligation. We hypothesized a priori that the CA diastolic flow relative to LV output would increase after PDA ligation and that low flow was associated with impaired LV performance.

\section{Study Population}

Premature infants born at less than 32 weeks of gestation who underwent PDA ligation at the Hospital for Sick Children (Toronto, ON, Canada) from July 2006 to March 2007 were eligible for enrollment. Newborns with congenital heart disease, except for a patent foramen ovale, were excluded from the present study. The study received ethics approval, a priori, from the institutional research ethics board, and the parents provided parental consent. 


$$
\begin{aligned}
& \text { Abbreviations and Acronyms } \\
& \text { CA }=\text { coronary artery } \\
& \text { hsPDA }=\text { hemodynamically significant patent } \\
& \text { ductus arteriosus } \\
& \text { LV }=\text { left ventricular } \\
& \text { LVO }=\text { left ventricular output } \\
& \text { NICU }=\text { neonatal intensive care unit } \\
& \text { PDA }=\text { patent ductus arteriosus } \\
& \text { PWD }=\text { pulse wave Doppler } \\
& \text { SMA }=\text { superior mesenteric artery } \\
& \text { VTI }=\text { velocity time integral } \\
& \text { VTI }_{\text {dias }}=\text { CA diastolic VTI }
\end{aligned}
$$

\section{hsPDA Diagnosis}

hsPDA was clinically suspected by certain characteristic features (eg, murmur, wide pulse pressure, bounding pulses, and/or hyperdynamic precordium) or early-onset systemic hypotension. Comprehensive 2-dimensional (2D) echocardiography was performed on all patients to quantify ductal characteristics before deciding on surgical ligation. The hsPDA was defined by a transductal diameter $>1.5 \mathrm{~mm}$ with unrestrictive $(<1 \mathrm{~m} / \mathrm{s})$ left to right transductal flow on pulse wave Doppler (PWD) and clinical features of pulmonary overcirculation and/or systemic hypoperfusion. ${ }^{6}$

\section{Medical Treatment}

The usual medical practice at referring institutions consisted of fluid restriction and nonsteroidal anti-inflammatory treatment. Two courses ( 6 doses of $0.2 \mathrm{mg} / \mathrm{kg}$ at 12-hour intervals) of indomethacin were administered, unless adverse effects or contraindications prevented it.

\section{Referral Process}

The study population included neonates referred from 2 neighboring inborn tertiary units and neonates from the study site, which is an outborn center. Neonates with hsPDA who either failed to respond to medical management or in which indomethacin was contraindicated were considered suitable for duct ligation. The decision to perform PDA ligation was made by a dedicated neonatologist-led PDA ligation team. All neonates undergoing PDA ligation underwent comprehensive echocardiography by a member of the team to define the size of the PDA and the magnitude of the transductal shunt according to the degree of pulmonary overcirculation and/or systemic hypoperfusion. Only neonates with a ductus of sufficient size and high-order magnitude of flow, as adjudicated by echocardiographic markers and the director of the PDA ligation team, were referred for PDA ligation. PDA ligation was not performed in the first 7 days of life in any neonate, because of concern regarding reperfusion intraventricular hemorrhage. All neonates were admitted at least 6 hours before the procedure.

\section{Preoperative Stabilization}

All patients had a hemoglobin level greater than $100 \mathrm{~g} / \mathrm{L}$, platelet count greater than $100,000 \mathrm{~mm}^{3}$, and international normalized ratio of 1 to 1.6 before surgical intervention. Neonates on high-frequency mode were switched to conventional ventilation at least 6 hours before surgery and enteral feeds withheld for similar duration. All neonates were transported from the referring hospital to the NICU at the surgical site by a dedicated neonatal transport team.

\section{Surgical Procedure}

The procedure was performed in the operating room by a cardiovascular surgeon, and all neonates were anesthetized with fentanyl and pancuronium. Newborns were placed in right lateral position and a left mid-axillary incision was made. The left lung was retracted, and the ductus arteriosus was mobilized. The ductus was then either ligated with sutures or metal clips according to the surgeon's preference, and an intercostal drain was placed prophylactically. Fluid boluses (normal saline) were administered in the case of hypotension or excessive fluid losses.

\section{Postoperative Care}

All neonates were transferred back to the NICU, and a morphine or fentanyl infusion was started. This was subsequently tapered for 6 to 24 hours depending on an objective assessment of pain using the Premature Infant Pain Profile score. ${ }^{7}$ A chest radiograph was performed on all infants within 1 hour after surgery to assess lung inflation and exclude air leaks. The usual practice was to monitor blood gases every 8 hour, depending on the postoperative course. During the study period, hypotension was defined using either of the following methods: (1) mean arterial pressure less than for the corrected gestational age or (2) systolic blood pressure less than the third percentile for corrected gestational age. The fluid boluses of normal saline $(10 \mathrm{~mL} / \mathrm{kg})$ and/or cardiotropes were administered to achieve normal blood pressure. If cardiotropic treatment was required, an infusion of dobutamine and/or dopamine at 5 to $10 \mu \mathrm{g} / \mathrm{kg} / \mathrm{min}$ was started and titrated to a maximum of $20 \mu \mathrm{g} / \mathrm{kg} / \mathrm{min}$, followed by the addition of epinephrine, if necessary. The selection of dobutamine or dopamine was at the discretion of the attending neonatologist who remained unaware of the results of the echocardiogram. Adjunctive steroid therapy with hydrocortisone was considered after failure of at least 2 cardiotropic agents. The usual postoperative recovery period in the NICU was at least 24 hours.

\section{Data Collection}

Baseline neonatal characteristics, details of the antenatal course, and delivery and resuscitation and preligation morbidities were recorded. Clinical and 2D echocardiographic characteristics of the hsPDA, dosage and duration of indomethacin treatment, and rationale for surgical intervention were reviewed. Physiologic indexes of cardiorespiratory stability (eg, heart rate, arterial pressure, oxygen saturation, and temperature) were extracted from the electronic patient chart preoperatively and at 1, 4, 8, 12, and 24 hours after the procedure. The mean arterial pressure was measured directly using a transducer connected to an indwelling catheter. The mean airway pressure and oxygenation index were monitored at these points, as surrogates of the degree of respiratory support and the efficacy of oxygenation. The oxygenation index was calculated according to the oxygenation index $=\left[\left(\right.\right.$ mean airway pressure $\left.\left./ \mathrm{PaO}_{2}\right) \times \mathrm{FiO}_{2} \times 100 \%\right]$, where $\mathrm{PaO}_{2}$ is the partial pressure of oxygen and $\mathrm{FiO}_{2}$ is the fractional inspired oxygen. Ventilation index was calculated as the [respiratory rate $\times(\mathrm{PIP}-$ $\left.\mathrm{PEEP}) \times \mathrm{PaCO}_{2}\right] / 1000$, where PIP is the positive inspired pressure, PEEP is the positive end-expiratory pressure, and $\mathrm{PaCO}_{2}$ is the partial pressure of arterial carbon dioxide. The need for fluid boluses or blood transfusion, and the amount of cardiotropic support was also recorded during the same period.

\section{D Echocardiography}

2D echocardiography was performed preoperatively (within 2 hours) and at 1, 8, and 24 hours after surgical intervention. 2D echocardiography was performed by a single operator (A.S.) using the Vivid 7 advantage cardiovascular ultrasound system (GE Medical Systems, Milwaukee, Wisconsin) with a 7.5- to 10-MHz, high-frequency phased array transducer probe. All images were saved to an optical disk for off-line analysis. Standard M-mode, 2D, PWD, continuous wave Doppler, and color Doppler evaluations were performed.

\section{CA Indexes}

The left anterior descending (LAD) CA was interrogated, because it is the main vessel supplying blood to the left ventricle and because previous 

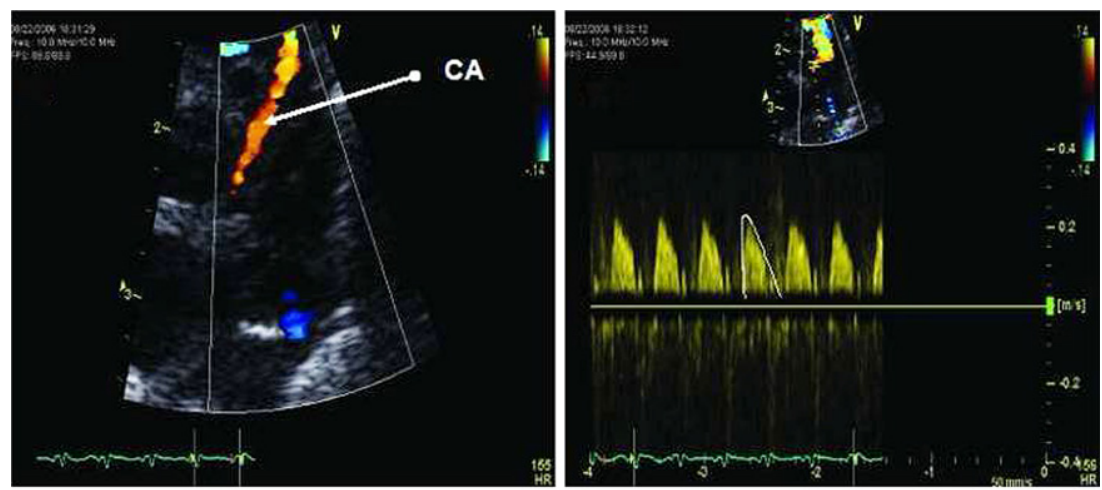

A

B

FIGURE 1. A, Color flow Doppler interrogation of left anterior descending coronary artery (CA, white arrow) from parasternal long axis approach. $\mathrm{B}$, Pulse-wave Doppler interrogation of coronary artery reveals predominant diastolic flow from which velocity time integral was traced (white triangulated line).

studies of term infants have shown $96 \%$ measurement feasibility. ${ }^{8}$ The left CA was best identified from the standard parasternal short-axis view using color Doppler, and the LAD CA branch is seen by moving the transducer down 1 or 2 intercostal spaces, rotating it clockwise, and angling it superiorly. The CA internal dimensions were measured at end-diastole just distal to its opening at the aorta with the calipers applied to the endothelial border. For color Doppler flow analysis, the scale was adjusted to an upper limit of 15 to $30 \mathrm{~cm} / \mathrm{s}$, allowing low-velocity signals to be identified. After identifying the CAs using 2D and color flow mapping, the PWD sample gate was placed over the LAD CA distal to the bifurcation and the blood flow signal was obtained, keeping the angle of the Doppler beam as parallel as possible to the direction of flow $\left(<15^{\circ}\right.$ in all recordings). Once a PWD trace was obtained, the CA diastolic velocity time integral $\left(\mathrm{VTI}_{\text {dias }}\right)$ was calculated by tracing the area under the curve (Figure 1) from an average of 5 consecutive beats. Because the predominant flow per minute in CAs is in diastole, calculations of flow were done using the following formula: CA flow $=\left(\mathrm{VTI}_{\text {dias }} \times\right.$ heart rate $\times$ CA cross-sectional area $) /$ body weight. The CA flow/LV output (LVO) ratio was then calculated to take into account the influence of LVO on coronary blood flow.

\section{Performance/End-Organ Perfusion}

The LV systolic (eg, fractional shortening, LVO) and diastolic performance (E and A wave peak velocity, E/A ratio, isovolumic relaxation time) were measured at each evaluation point. ${ }^{8,9}$ The indexes of systemic blood flow (eg, superior vena cava flow, LVO) were estimated according to published methods. ${ }^{10}$ The proximal segment of the superior mesenteric artery (SMA) was imaged in the long-axis sagittal plane. ${ }^{11} \mathrm{PWD}$ was then applied to the region of interest and trends in SMA velocities were compared before and 1, 8, and 24 hours after ligation. The SMA resistance index was calculated at each point using the formula: $\left(\mathrm{SMA}_{\text {syst }}-\mathrm{SMA}_{\mathrm{dias}}\right) /$ $\mathrm{SMA}_{\text {syst }}$, where syst is systolic and dias is diastolic.

\section{Ductus Arteriosus Evaluation}

The transductal diameter, flow direction (left-to-right or bidirectional), and flow patterns (unrestrictive vs restrictive) were estimated at the narrowest end of the ductus arteriosus.

\section{Pulmonary Hemodynamics}

The right ventricular systolic pressure was calculated using a 4-chamber approach according to the following calculation: $[(4 \times$ peak tricuspid artery velocity $)^{2}+5$ ]. The pulmonary artery was interrogated from an oblique long-axis approach, and PWD was applied to obtain the flow waveform and trace the VTI. The right ventricular output was then calculated as
$[($ pulmonary artery VTI $\times$ heart rate $) \times$ pulmonary artery crosssectional area)]/body weight.

\section{Laboratory Testing}

Arterial blood gas and plasma lactate testing was performed at baseline and 1,8 , and 24 hours after surgical ligation.

\section{Outcome Measures}

The primary outcome was the postoperative CA diastolic flow/LVO ratio. The secondary outcomes were the postoperative arterial pressure, systemic blood flow (LVO), systolic (fractional shortening) and diastolic myocardial performance (E wave and E/A ratio) and the need for cardiotropes. The frequency of impaired LV performance, defined by a LVO of less than $170 \mathrm{~mL} / \mathrm{kg} / \mathrm{min}$ or systolic blood pressure less than the third percentile, was documented.

\section{Statistical Analysis}

Descriptive statistics were used to characterize the baseline clinical and echocardiography characteristics. Analysis of covariance (ANCOVA) testing was used to analyze the serial changes over time in the indexes of LV diastolic performance ( $\mathrm{E}$ wave, A wave, and isovolumic relaxation time) and systolic performance (CA flow/LVO ratio, $\mathrm{VTI}_{\text {dias }}$, and LVO). Continuous variables were analyzed using the $t$ test or Mann-Whitney $U$ test for parametric and nonparametric data sets, respectively. The chi-square and Fisher exact test were used to analyze the categorical variables. Linear regression analysis was performed to investigate the relationship between the $\mathrm{VTI}_{\text {dias }}$ and systolic blood pressure ( 8 hours), diastolic performance ( 1 hour), and the need for cardiotropic support. Significance was set at $P<.05$. Subgroup analysis was performed between neonates who had received cardiotropes and those who had not. Because this was a hypothesisgenerating pilot study, a sample size of convenience was chosen a priori to recruit 20 infants.

\section{RESULTS}

\section{Neonatal Characteristics}

A total of 25 neonates underwent PDA ligation during the study period. However, 5 neonates were not enrolled because of parental refusal of consent. Thus, 20 infants were studied whose mean gestational age and median weight at birth was $26.3 \pm 0.7$ weeks and $780 \mathrm{~g}$ (range, 570-2400), respectively. The mean transductal diameter at ligation was $3.2 \pm 0.4 \mathrm{~mm}$, 
TABLE 1. Baseline neonatal demographics, attributable morbidities, and characteristics of ductus arteriosus $(n=20)$

\begin{tabular}{lc}
\hline \multicolumn{1}{c}{ Neonatal characteristics } & Value \\
\hline Age at PDA clinical detection (days) & $5.1 \pm 3.2$ \\
Age at 2D echocardiographic detection (days) & $9.6 \pm 6.9$ \\
Indomethacin doses (n) & $4.2 \pm 3.2$ \\
Weight at intervention (g) & $1160 \pm 603$ \\
Age at surgical intervention (days) & $26.1 \pm 10.4$ \\
Corrected gestational age at intervention (days) & $29.2 \pm 2.0$ \\
Duration of surgery (min) & $72.5(65-80)$ \\
Preoperative neonatal morbidity (n) & \\
$\quad$ Respiratory distress syndrome & 15 \\
Intraventricular hemorrhage & 8 \\
$\quad$ Necrotizing enterocolitis & 6 \\
Preoperative co-intervention (n) & \\
Prophylactic indomethacin & 2 \\
Fluid restriction & 13 \\
Diuretics & 8 \\
Cardiotropes & 2 \\
Postoperative complications (n) & \\
Need for HFOV & \\
Cardiotropic support & 7 \\
\hline
\end{tabular}

Data are presented as mean $\pm \mathrm{SD}$ or median (interquartile range). $P D A$, Patent ductus arteriosus; $2 D, 2$-dimensional; $H F O V$, high-frequency oscillatory ventilation.

and all infants had an unrestrictive left-to-right shunt (Table 1) at the intervention. The postoperative course was characterized by a trend toward a decreased systolic arterial pressure and increased oxygenation index (Table 2).

\section{CA Perfusion}

PDA ligation was followed by a nonsignificant trend toward increased total CA flow at 1 hour. The CA diameter was unchanged after surgery with a pre- and postintervention value of $0.11 \pm 0.01$. The $\mathrm{CA}$ diastolic flow/LVO ratio showed interval improvement, reaching a peak 1 hour after surgical intervention (Figure 2). We therefore examined the proportionate changes in CA flow and LVO at 1 hour versus the preoperative values and found a significant increase $(P<.001)$ in median CA flow $(54.4 \%$; range, $24.1-$ $78.7 \%$ ) compared with $\mathrm{LVO}$, which was characterized by a net reduction in flow (median, $-52.8 \%$; range $-40 \%$ to $-65.9 \%$ ). A lower preoperative $\mathrm{VTI}_{\text {dias }}$ was associated with a lower systolic arterial pressure at 8 hours ( $r=0.59, P=.05)$ and 12 hours $(r=0.52, P=.04)$, greater fractional inspired oxygen at 24 hours $(r=0.52, P=.003)$ and lower LVO $(r=0.58, P=.03)$ at 1 hour after ligation (Table 3). We found no association, however, between the $\mathrm{VTI}_{\text {dias }}$ and either the mean or diastolic arterial pressure at any point (data not shown).

\section{Myocardial Performance}

LV systolic performance. PDA ligation was followed by a reduction in fractional shortening and mean velocity of circumferential fiber shortening $(P<.001$, ANCOVA), which were greatest 8 hours after surgical intervention (Table 4). A significant decrease in LVO $(P<.001$, ANCOVA) was identified that peaked at 8 hours.

LV diastolic performance. Sustained reductions in E wave $(P=.002$, ANCOVA $)$, A wave $(P=.02$, ANCOVA $)$, and peak velocity from 1 hour onward were seen. These changes were temporally associated with a sustained elevation of the isovolumic relaxation time $(P<.001$, ANCOVA), and a reduction in the LV end-diastolic diameter and left atrial/aortic ratio, reflective of an altered left heart preload, was also seen. An association between the baseline CA diastolic flow and 1-hour E wave velocity $(r=0.64$, $P=.03)$ and the E/A wave velocity ratio $(r=0.63$, $P=.01$ ) was identified (Table 3).

Pulmonary hemodynamics. PDA ligation resulted in a significant decrease in RVO $(P<.001$ ANCOVA), although no changes were seen in the right ventricular systolic pressure.

End-organ flow. PDA ligation was followed by an increase in peak diastolic velocity in the SMA with a corresponding decline in pulsatility index $(P=.003)$. The diastolic flow in the SMA was either absent or reversed in all patients before surgical intervention.

Cardiotropic support. Of the 20 patients, 8 received cardiotropic support; 2 received dopamine, 5 received dobutamine, 1 received both, and 1 received hydrocortisone. The median point of the initiation of cardiotropes was 8 hours, coinciding with the lowest point of systolic arterial pressure and peak oxygenation index, findings comparable with our previous publication on the subject. ${ }^{5}$ Neonates who received cardiotropes underwent surgical ligation at an earlier postnatal age, were more likely to develop postoperative cardiorespiratory instability, and develop lower 1-hour $\mathrm{VTI}_{\text {dias }}$, 1- and 8-hour LVO, and 8-hour fractional shortening (Table 5). Finally, the proportionate change in $\mathrm{VTI}_{\text {dias }}$ between the preoperative and 1-hour values differed $(P=.002)$. The levels decreased in those neonates who required cardiotropes $(-40.8 \%$; range, $-49.7 \%$ to $-15.2 \%)$ but increased in those who did not $(23.7 \%$; range, $7.5 \%$ to $76.2 \%$ ).

\section{DISCUSSION}

Surgical ligation of the ductus arteriosus was associated with impairment in LV systolic performance and a low cardiac output state, as previously reported. In the present study, we demonstrated changes in CA diastolic flow, suggesting it might be compromised in neonates with hsPDA. Perioperative Doppler evaluations of CA blood flow might facilitate earlier identification of neonates at greatest risk of postligation hemodynamic instability.

\section{Coronary Perfusion and hsPDA}

The ductus arteriosus acts as a low-vascular resistance circuit, not too dissimilar to the placenta in fetal life. 
TABLE 2. Perioperative indexes of cardiorespiratory stability after PDA ligation

\begin{tabular}{|c|c|c|c|c|c|c|c|}
\hline \multirow[b]{2}{*}{ Variable } & \multirow[b]{2}{*}{ Baseline } & \multicolumn{5}{|c|}{ Postoperatively (hr) } & \multirow[b]{2}{*}{$P$ value } \\
\hline & & 1 & 4 & 8 & 12 & 24 & \\
\hline \multicolumn{8}{|l|}{$\mathrm{AP}(\mathrm{mm} \mathrm{Hg})$} \\
\hline Systolic & $55.6 \pm 11.8$ & $57.4 \pm 13.3$ & $53.3 \pm 12.6$ & $45.6 \pm 11.3$ & $50.6 \pm 16.2$ & $55.4 \pm 11.5$ & .09 \\
\hline Diastolic & $27.0 \pm 10.1$ & $33.7 \pm 11.6$ & $30.3 \pm 9.6$ & $31.6 \pm 9.6$ & $28.1 \pm 11.9$ & $32.1 \pm 8.5$ & NS \\
\hline Mean & $37.1 \pm 8.9$ & $42.4 \pm 11.9$ & $9.0 \pm 10.9$ & $39.2 \pm 11.3$ & $35.5 \pm 15.4$ & $40.7 \pm 9.3$ & NS \\
\hline Respiratory rate (breaths/min) & $49(44-54)$ & $40(35-45)$ & $42(40-47)$ & $40(36-46)$ & $45(42-52)$ & $44(41-49)$ & NS \\
\hline $\mathrm{MAP}\left(\mathrm{cm} \mathrm{H}_{2} \mathrm{O}\right)$ & $8.1(7.4-11)$ & $9.6(8.2-10)$ & $9(8.3-9.2)$ & $9.6(8.3-11.5)$ & $9.3(8.1-11)$ & $9.6(9-11.5)$ & NS \\
\hline Ventilation index & $22.6 \pm 6.7$ & & & $21.9 \pm 7.5$ & & $22.5 \pm 6.5$ & NS \\
\hline $\mathrm{FiO}_{2}(\%)$ & $30(24-41)$ & $35(30-45)$ & $30(27-36)$ & $43(31-50)$ & $38(30-45)$ & $35(27-42)$ & NS \\
\hline OI & $5.8(4.5-9)$ & & & $9.1(7-12)^{*}$ & & $7.1(5.5-12)$ & .04 \\
\hline Arterial $\mathrm{pH}$ & $7.33 \pm 0.08$ & & & $7.3 \pm 0.06$ & & $7.34 \pm 0.07$ & NS \\
\hline Base deficit & $-0.3 \pm 3.8$ & & & $0.4 \pm 3.9$ & & $-0.6 \pm 4.0$ & NS \\
\hline Heart rate (bpm) & $155 \pm 12$ & $149 \pm 14$ & $158 \pm 12$ & $150 \pm 13$ & $158 \pm 11$ & $152 \pm 16$ & NS \\
\hline
\end{tabular}

Data are presented as mean \pm SD or median (interquartile range). $A P$, Arterial pressure; $N S$, not significant; $M A P$, mean airway pressure; $\mathrm{FiO}_{2}$, fractional inspired oxygen; $O I$, oxygen index. $* P<.05$ vs baseline value.

Because the shunt in these infants is from the systemic circulation to pulmonary, the left ventricle contracts against a cumulative pulmonary and systemic vascular resistance, which is lower than expected. The clinical consequences include a wide pulse pressure and low-diastolic arterial pressure. The evidence of end-organ hypoperfusion can be inferred from Doppler evaluation of the mesenteric, cerebral, or renal artery flow patterns. Specifically, reversal or absence of diastolic perfusion is pathognomonic of hsPDA. ${ }^{12,13}$ An increase in LV output is commonly seen owing to the cumulative effects of increased left heart preload and a lower left ventricle-exposed vascular resistance. The principal determination of coronary perfusion pressure is aortic diastolic pressure, which is low in neonates with hsPDA. This can lead to compromised coronary perfusion and consequential impairment in myocardial

\section{CA : LVO ratio}

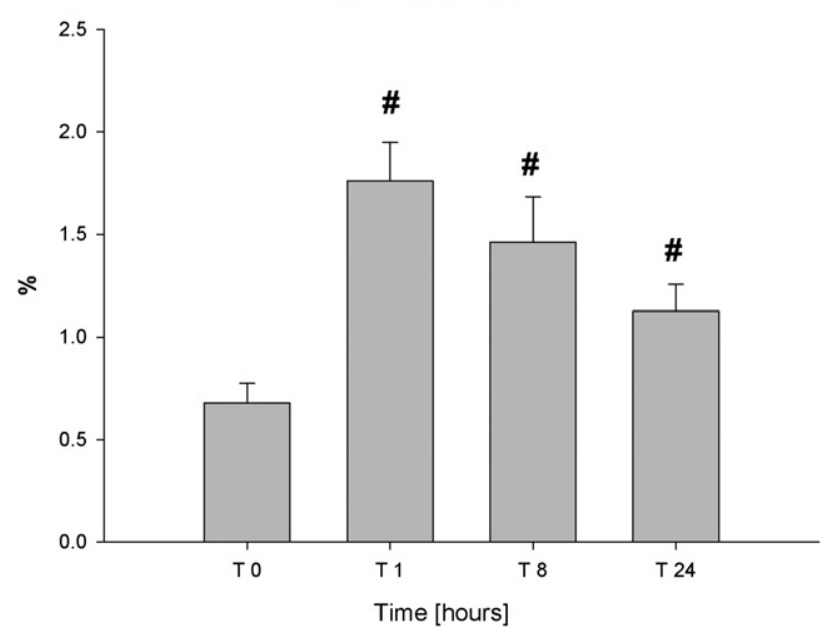

FIGURE 2. Changes in coronary artery $(C A)$ flow relative to left ventricular output $(L V O)$ before and after surgical ligation of ductus arteriosus. $\# P<.05$ versus baseline. performance with an expectant reduction in cardiac output. We believe the increase in cardiac output, seen in our cohort, reflects the natural compensatory response of the myocardium to impairment in pulmonary and systemic hemodynamics. By the time of surgical intervention, the cardiac output has reached a satisfactory compensatory state, with little obvious impairment in arterial pressure or other markers of the adequacy of systemic blood flow. When subjected to increased vascular resistance and altered cardiac loading conditions after surgical ligation, the left ventricle is unable to compensate.

We identified a $40 \%$ increase in CA flow after ductal closure, but this did not reach statistical significance. On normalization of the diastolic arterial pressure, the CA perfusion pressure should increase and augment the CA flow. The postoperative decrease in LVO, however, can influence changes in CA flow, independent of the CA perfusion pressure. We therefore chose to index the CA flow to the LVO and identified a significant increase in proportionate the CA flow 1 hour after PDA ligation. Because the timing of this change coincided temporally with a decrease in LVO

TABLE 3. Regression equations for variables associated with baseline and 1-hour coronary artery diastolic velocity time integral

\begin{tabular}{lrlrl}
\hline $\begin{array}{c}\text { Independent } \\
\text { variable (y) }\end{array}$ & Intercept & $\begin{array}{c}\text { Slope of dependant } \\
\text { variable (x) }\end{array}$ & $\boldsymbol{r}$ value & $\begin{array}{c}\boldsymbol{P} \\
\text { value }\end{array}$ \\
\hline $\mathrm{SAP}(\mathrm{hr})$ & & & & \\
8 & 38.9 & $+5.2 \mathrm{VTI}_{\text {Base }}$ & 0.59 & .05 \\
12 & 35.7 & $+6.3 \mathrm{VTI}_{\text {Base }}$ & 0.52 & .04 \\
$\mathrm{FiO}_{2}$ at $24 \mathrm{hr}$ & 46.5 & $-2.7 \mathrm{VTI}_{\text {Base }}$ & 0.52 & .003 \\
$\mathrm{E} \mathrm{wave} \mathrm{V}_{\text {max }}$ at $1 \mathrm{hr}$ & 0.3 & $+0.05 \mathrm{VTI}_{\text {Base }}$ & 0.64 & .03 \\
$\mathrm{E} / \mathrm{A}$ wave at $1 \mathrm{hr}$ & 0.4 & $+0.1 \mathrm{VTI}_{\text {Base }}$ & 0.63 & .01 \\
LVO at $1 \mathrm{hr}$ & 122.9 & $+40.6 \mathrm{VTI}_{\text {Base }}$ & 0.58 & .03 \\
\hline
\end{tabular}

$S A P$, Systolic arterial pressure; $V T_{\text {base }}$, velocity time integral at baseline; $\mathrm{FiO}_{2}$, fractional inspired oxygen; $E$ wave, passive filling phase; $V_{\max }$, peak velocity of flow; $A$ wave, active filling phase; $L V O$, left ventricular output. 
TABLE 4. Two-dimensional echocardiography indexes of hemodynamic stability after PDA ligation

\begin{tabular}{|c|c|c|c|c|c|}
\hline Variable & Baseline & 1 hours & 8 hours & 24 hours & $P$ value \\
\hline LA/Ao ratio & $2.1 \pm 0.4$ & $1.8 \pm 0.4$ & $1.7 \pm 0.3^{*}$ & $1.8 \pm 0.4^{*}$ & .04 \\
\hline LVEDD (cm) & $1.5(1.3-1.7)$ & $1.2(1.0-1.4)$ & $1.2(1.0-1.3)^{*}$ & $1.2(1.1-1.5)^{*}$ & .02 \\
\hline IVRT (ms) & $27.5 \pm 9.4$ & $47.2 \pm 15.2^{*}$ & $45.6 \pm 16.2 *$ & $47.0 \pm 13.5^{*}$ & $<.001$ \\
\hline E wave $V_{\max }(\mathrm{cm} / \mathrm{s})$ & $0.64(0.53-0.78)$ & $0.39(0.3-0.47)^{*}$ & $0.41(0.36-0.53)^{*}$ & $0.52(0.38-0.6)$ & .001 \\
\hline A wave $V_{\max }(\mathrm{cm} / \mathrm{s})$ & $0.83 \pm 0.14$ & $0.66 \pm 0.23^{*}$ & $0.62 \pm 0.18^{*}$ & $0.71 \pm 0.19$ & .02 \\
\hline $\mathrm{E}$ wave/A wave ratio & $0.9 \pm 0.1$ & $0.6 \pm 0.2 *$ & $0.7 \pm 0.2 *$ & $0.6 \pm 0.2$ & $<.001$ \\
\hline LVO (mL/min/kg) & $502(385-724)$ & $260(143-368)^{*}$ & $282(185-381)^{*}$ & $370(270-417)$ & $<.001$ \\
\hline Aortic $\mathrm{V}_{\max }(\mathrm{cm} / \mathrm{s})$ & $1.2 \pm 0.2$ & $0.7 \pm 0.2 *$ & $0.7 \pm 0.1^{*}$ & $0.9 \pm 0.2 *$ & $<.001$ \\
\hline Superior vena cava VTI $(\mathrm{cm})$ & $10.3(8.6-12.8)$ & $10.6(8.8-11.9)$ & $8.2(7.3-11)$ & $9.5(8-11.6)$ & NS \\
\hline CA flow $(\mathrm{mL} / \mathrm{min} / \mathrm{kg})$ & $2.4 \pm 1.2$ & $3.3 \pm 1.2$ & $3.1 \pm 1.5$ & $3.1 \pm 1.5$ & .09 \\
\hline $\mathrm{RVO}(\mathrm{mL} / \mathrm{min} / \mathrm{kg})$ & $638(463-930)$ & $269(204-430)^{*}$ & $285(201-364)^{*}$ & $402(228-462)^{*}$ & .005 \\
\hline RV systolic pressure (mm Hg) & $6.9(5.5-11.4)$ & $6.4(5.7-11.7)$ & $6.8(5.7-10.7)$ & $9(5.8-15)$ & NS \\
\hline Superior mesenteric systolic $V_{\max }(\mathrm{cm} / \mathrm{s})$ & $0.5 \pm 0.1$ & $0.4 \pm 0.2$ & $0.4 \pm 0.2$ & $0.6 \pm 0.2$ & .07 \\
\hline Superior mesenteric diastolic $\mathrm{V}_{\max }(\mathrm{cm} / \mathrm{s})$ & $0.06(0-0.15)$ & $0.17(0.14-0.19)^{*}$ & $0.14(0.13-0.16)$ & $0.15(0.14-0.16)$ & .02 \\
\hline SMA pulsatility index & $0.89(0.72-1)$ & $0.61(0.54-0.63)^{*}$ & $0.68(0.55-0.73)^{*}$ & $0.76(0.68-0.78)$ & .003 \\
\hline
\end{tabular}

Data are presented as mean $\pm \mathrm{SD}$ or median (interquartile range). $L A$, Left atrium; $A o$, aorta; $L V E D D$, left ventricular end-diastolic dimension; IVRT, isovolumic relaxation time; $V_{\max }$, peak velocity of flow; $L V O$, left ventricular output; $V T I$, velocity time integral; $C A$, coronary artery; $R V O$, right ventricular output; $R V$, right ventricular; $S M A$, superior mesenteric artery. $* P<.05$ vs baseline value

and was followed by a subsequent cumulative decrease in the net CA flow, we believe this reflects a real increase in net CA flow at 1 hour and not just a mathematical correction. The temporal relationship of these changes in coronary flow to normalization of SMA flow suggests that CA flow can also be compromised by hsPDA. Because CA perfusion is dependent on preductal blood flow, altered CA flow might suggest a more pathologic hemodynamic state.

El-Khuffash and Molloy ${ }^{4}$ reported elevated plasma troponin levels in a cohort of premature infants with hsPDA that normalized after therapeutic intervention, further supporting our hypothesis of compromised CA perfusion. In addition, Way and colleagues ${ }^{3}$ reported significant ST depression on the electrocardiogram in $9.5 \%$ of preterm infants with symptomatic PDA. The consequences of intermittent or sustained CA hypoperfusion might include acute impairment in myocardial performance or impaired responsiveness of the myocardium to altered loading conditions, such as is seen after PDA ligation. In physiologic terms, the CA diastolic blood flow has the potential to be a sensitive marker of ductal significance and myocardial health.

\section{Coronary Perfusion and Myocardial Performance}

Previously, we reported impaired LV systolic performance in patients weighing less than $1000 \mathrm{~g}$, which is most likely related to alterations in cardiac loading conditions. ${ }^{5}$ In the present study, we have demonstrated an association between preoperative $\mathrm{VTI}_{\text {dias }}$ and postoperative impairment in diastolic performance, low LVO, low systolic arterial pressure, and oxygen requirement at 24 hours. $\mathrm{VTI}_{\text {dias }}$ at 1 hour postoperatively was also lower in infants who required cardiotropes. Oskarsson and Pesonen ${ }^{8}$ studied flow in the LAD CA of healthy term neonates. Specifically, they identified a correlation between CA flow and transmitral early velocity (E wave) and the aortic VTI. ${ }^{8}$ Coronary blood flow in the newborn is closely linked to myocardial oxygen consumption, which is assumed to be related to cardiac work and the rate-pressure product. ${ }^{14}$ Although no correlation was found between flow in the LAD CA and either heart rate or blood pressure, a linear correlation with aortic VTI suggests that an increased myocardial work associated with increased LV output demands an augmentation in LAD CA flow. The correlation between the baseline diastolic coronary flow and postoperative need for cardiotropes suggests increased susceptibility to myocardial dysfunction; this might relate to chronic myocardial ischemia as highlighted earlier. This subgroup of neonates who received cardiotropes was younger at surgical intervention; postoperatively, they had a lower systolic arterial pressure at 8 hours, lower arterial $\mathrm{pH}$ and base deficit, and increased fractional inspired oxygen at 24 hours. The echocardiographic evaluation demonstrated a lower LVO at 1 and 8 hours, a lower CA VTI at 1 hour, and lower fractional shortening at 8 hours, changes that coincided with the clinical deterioration described. In addition, a low perioperative diastolic coronary flow was associated with the oxygen requirements, possibly owing to elevated left atrial pressure and pulmonary edema. Although the oxygen requirements did increase, this did not reach statistical significance, which might reflect the low sample size. These data suggest that in addition to the clinical and echocardiographic criteria used for staging the hemodynamic significance of the ductus arteriosus, ${ }^{15,16}$ perioperative CA flow might facilitate earlier identification of neonates at a greater risk of developing postoperative cardiovascular compromise. It is not possible to determine from this study whether a threshold level of CA flow exists below which myocardial ischemia occurs. Likely, a critical 
TABLE 5. Comparative evaluation of neonates who did and did not received cardiotropic support

\begin{tabular}{|c|c|c|c|}
\hline Variable & $\begin{array}{c}\text { Cardiotropes } \\
(\mathbf{n}=\mathbf{8})\end{array}$ & $\begin{array}{l}\text { No cardiotropes } \\
\quad(\mathbf{n}=12)\end{array}$ & $\begin{array}{c}P \\
\text { value }\end{array}$ \\
\hline Gestational age (wk) & $24.8 \pm 1.6$ & $25.6 \pm 1.9$ & NS \\
\hline Birth weight (g) & $794 \pm 149$ & $758 \pm 181$ & NS \\
\hline Weight at surgery (g) & $961 \pm 221$ & $972 \pm 111$ & NS \\
\hline Age at surgery (days) & $18.8 \pm 6.8$ & $30.6 \pm 8.4$ & .02 \\
\hline Transductal diameter (mm) & $2.9(2.7-3.0)$ & $3.0(2.8-3.2)$ & NS \\
\hline \multicolumn{4}{|l|}{ Preoperative status } \\
\hline Heart rate (bpm) & $158 \pm 12$ & $155 \pm 12$ & NS \\
\hline Systolic AP (mm Hg) & $52 \pm 10$ & $55 \pm 13$ & NS \\
\hline Diastolic AP (mm Hg) & $26 \pm 8$ & $25 \pm 10$ & NS \\
\hline $\mathrm{FiO}_{2}(\%)$ & $39 \pm 17$ & $35 \pm 12$ & NS \\
\hline Arterial $\mathrm{pH}$ & $7.28(7.26-7.31)$ & $7.34(7.28-7.43)$ & NS \\
\hline \multicolumn{4}{|l|}{ Postoperative status } \\
\hline Heart rate $(\mathrm{bpm})$ & $158 \pm 12$ & $155 \pm 12$ & NS \\
\hline Systolic AP at $8 \mathrm{hr}(\mathrm{mm} \mathrm{Hg})$ & $41 \pm 12$ & $56 \pm 10$ & .02 \\
\hline Diastolic AP at $8 \mathrm{hr}(\mathrm{mm} \mathrm{Hg})$ & $28 \pm 9$ & $32 \pm 7$ & NS \\
\hline Maximum MAP $\left(\mathrm{cm} \mathrm{H}_{2} \mathrm{O}\right)$ & $11.1 \pm 1.5$ & $10.1 \pm 1.9$ & NS \\
\hline $\mathrm{FiO}_{2}$ at $24 \mathrm{hr}(\%)$ & $48 \pm 17$ & $35 \pm 7$ & .04 \\
\hline Minimum arterial $\mathrm{pH}$ & $7.26 \pm 0.07$ & $7.33 \pm 0.06$ & .09 \\
\hline Maximum base deficit & $-3.8 \pm 2.7$ & $1.3 \pm 2.1$ & .002 \\
\hline \multicolumn{4}{|l|}{ Postoperative echocardiography } \\
\hline $\begin{array}{l}\text { Fractional shortening } \\
\text { at } 8 \mathrm{hr}(\%)\end{array}$ & $26 \pm 8$ & $41 \pm 10$ & .01 \\
\hline $\mathrm{LVO}$ at $1 \mathrm{hr}(\mathrm{mL} / \mathrm{min} / \mathrm{kg})$ & $179 \pm 44$ & $331 \pm 101$ & .01 \\
\hline $\mathrm{LVO}$ at $8 \mathrm{hr}(\mathrm{mL} / \mathrm{min} / \mathrm{kg})$ & $185 \pm 29$ & $260 \pm 65$ & .02 \\
\hline CA VTI at $1 \mathrm{hr}$ & $1.9 \pm 1.2$ & $3.41 \pm 1$ & .01 \\
\hline CA VTI $\times$ heart rate at $1 \mathrm{hr}$ & $284 \pm 175$ & $496 \pm 168$ & .03 \\
\hline SMA diastolic $\mathrm{V}_{\max }$ & $0.04 \pm 0.08$ & $0.12 \pm 0.06$ & .08 \\
\hline
\end{tabular}

$A P$, Arterial pressure; $\mathrm{FiO}_{2}$, fractional inspired oxygen; $\mathrm{MAP}$, mean airway pressure; $L V O$, left ventricular output; $C A$, coronary artery; VTI, velocity time integral; SMA, superior mesenteric artery; $V_{\max }$, peak velocity of flow.

duration of exposure to low CA flow exists, after which the myocardium is susceptible to afterload-related impairment in performance. Both questions require a prospective evaluation.

\section{Performance-Additional Insights}

PDA ligation was associated with a significant reduction in LV fractional shortening and mean velocity of circumferential fiber shortening, temporally related to an increase in left ventricle-exposed vascular resistance, such as was seen in our previous study and has been reported by others. ${ }^{5}$ In the present study, we demonstrated that PDA ligation was followed by a reduction in the $\mathrm{E}$ and $\mathrm{A}$ wave peak velocity and an increase in the isovolumic relaxation time. Normal transmitral flow is typically biphasic and characterized by an $\mathrm{E}$ wave phase, reflecting early passive transmitral filling, and a later A wave phase, reflecting active left atrial contraction. Developmentally, the E/A wave ratio is approximately 0.7 at 16 weeks, which is thought to reflect myocardial immaturity and impaired myocardial compliance and increases to $\sim 0.9$ to $1: 1$ around term gestation. In the presence of hsPDA in which pulmonary overcirculation is present, the passive component increases secondary to left heart pressure loading, leading to pseudonormalization of the E/A ratio $(>1.0)$ to an adult-like pattern. ${ }^{17}$ The isovolumic relaxation time is the period between closure of mitral valve and opening of the aortic valve and is typically shortened ( $<35$ seconds) in the situation of pressure or volume loading of the left atrium. The changes in the transmitral velocities after PDA ligation might relate to reduced left heart preload or impaired myocardial compliance and diastolic dysfunction, because they coincided with a sustained elevation in the isovolumic relaxation time. The clinical relevance of these findings remains unknown, but because they preceded any clinical deterioration, they might also serve as early predictors of postoperative hemodynamic instability.

The present study had several limitations. First, operatordependant factors might have influenced the estimation of $\mathrm{CA}$ diameter. The $\mathrm{CA}$ diameter ranges from 0.8 to 1.2 $\mathrm{mm}$ in neonates; thus, small errors in measurement can cause significant error in the calculation of CA flow. The CAs move with each heart beat, and it can sometimes be difficult to maintain the sample volume of the PWD within the lumen of the vessel throughout systole and diastole. It is possible that the postoperative CA flow was modified by the choice of cardiotrope, acting through different pathways; however, the low event rate precluded a meaningful analysis. Additionally, it is possible that variability in physician practices and preferences related to the administration of crystalloid therapy or cardiotropic agents might have modified the hemodynamic trends, although no clear patterns emerged. There could be intraoperative hemodynamic changes that influence both CA blood flow and subsequent myocardial performance; however, we did not monitor these parameters during surgery. Reduced cerebral oxygen saturation, measured using near-infrared spectroscopy, was demonstrated during surgery. ${ }^{18}$ Since the completion of the present study a dedicated PDA ligation team has been created to standardize the triage and referral process for surgical intervention and to supervise the postoperative care to improve consistency in management.

\section{CONCLUSIONS}

The CA flow changes after PDA ligation, suggesting it is potentially compromised by a high order of magnitude ductal shunt. Its clinical relevance and value in determining the magnitude of the shunt requires prospective evaluation. In addition, CA perfusion might play an important contributory role in the etiopathogenesis of postligation hemodynamic instability. Lower perioperative diastolic coronary perfusion is associated with impaired postoperative myocardial systolic and diastolic performance, although a causal relationship could not be elicited. CA flow indexes might help facilitate earlier detection of neonates at greater risk of hemodynamic instability. The temporal relationship of surgical intervention to altered transmitral flow is 
noteworthy, providing a plausible rationale for the nature of postoperative oxygenation failure, pulmonary edema, and systemic hypoperfusion. Future studies should investigate the value of CA flow as a marker of hemodynamic significance in neonates with ongoing ductal patency and define the thresholds for therapeutic intervention.

\section{References}

1. Ellison RC, Peckham GJ, Lang GJP, Talner NS, Lerer TJ, Lin L, et al. Evaluation of the preterm infant for patent ductus arteriosus. Pediatrics. 1983;71:364-72.

2. Arnold G, Kosche F, Miessner E, Neitzert A, Lochner W. The importance of the perfusion pressure in the coronary arteries for the contractility and the oxygen consumption of the heart. Pflugers Arch Gesamte Physiol Menschen Tiere. 1968;299:339-56.

3. Way GL, Pierce JR, Wolfe RR, McGrath R, Wiggins J, Merenstein GB. ST depression suggesting subendocardial ischemia in neonates with respiratory distress syndrome and patent ductus arteriosus. J Pediatr. 1979;95:609.

4. El-Khuffash AF, Molloy EJ. Influence of a patent ductus arteriosus on cardiac troponin T levels in preterm infants. J Pediatr. 2008;153:350-3.

5. McNamara PJ, Teixera LS, Sehgal A. Patent ductus arteriosus ligation is associated with impaired left ventricular systolic performance in premature infants weighing less than 1000 g. J Thorac Cardiovasc Surg. 2010;140:150-7.

6. Kluckow M, Evans N. Early echocardiographic prediction of symptomatic patent ductus arteriosus in preterm infants undergoing mechanical ventilation. J Pediatr. 1995;127:774-9.

7. Stevens B, Johnston C, Pteryshen P, Taddio A. Premature infant pain profile: development and initial validation. Clin J Pain. 1996;12:13-22.
8. Oskarsson G, Pesonen E. Coronary blood flow in healthy neonates: effects of left ventricular function and mass. Pediatr Cardiol. 2004;25:11-6.

9. Silverman NH, Schiller NB. Cross sectional echocardiographic assessment of cardiac chamber size and ejection fraction in children. Ultrasound Med Biol. 1984;10:757-69.

10. Kluckow M, Evans N. Superior vena cava flow in newborn infants: a novel marker of systemic blood flow. Arch Dis Child Fetal Neonatal Ed. 2000;82: F182-7.

11. Bel FV, Zoeren DV, Schipper J, Gult GL, Baan J. Effect of indomethacin on superior mesenteric artery blood flow velocity in preterm infants. J. Pediatr. 1990; 116:965-70.

12. Shimada S, Kasai T, Konishi M, Fujiwara T. Effects of patent ductus arteriosus on left ventricular output and organ blood flows in preterm infants with respiratory distress syndrome treated with surfactant. J Pediatr. 1994;125:270-7.

13. Meyers RL, Alpan G, Lin E, Clyman RI. Patent ductus arteriosus, indomethacin, and intestinal distension: effects on intestinal blood flow and oxygen consumption. Pediatr Res. 1991;29:569-74.

14. White WB. Heart rate and the rate-pressure product as determinants of cardiovascular risk in patients with hypertension. Am J Hypertens. 1999;12:50S-5S.

15. McNamara PJ, Sehgal A. Towards rational management of the patent ductus arteriosus: the need for disease staging. Arch Dis Child Fetal Neonatal Ed. 2007; 92:F424-7.

16. Sehgal A, McNamara PJ. Echocardiographic markers of a haemodynamically significant ductus arteriosus. Eur J Pediatr. 2009;168:907-14.

17. Schmitz L, Stiller B, Koch H, Koehne P, Lange P. Diastolic left ventricular function in preterm infants with a patent ductus arteriosus: a serial Doppler echocardiography study. Early Hum Dev. 2004;76:91-100.

18. Lemmers PM, Molenschot MC, Evens J, Toet MC, van Bel F. Is cerebral oxygen supply compromised in preterm infants undergoing surgical closure for patent ductus arteriosus? Arch Dis Child Fetal Neonatal Ed. 2010;95:F429-34. 